\title{
A data review of airway management in patients with oral cavity or oropharyngeal cancer: a single-institution experience
}

\author{
Gang Zheng ${ }^{1 *}$ D, Lei Feng ${ }^{2}$ and Carol M. Lewis ${ }^{3}$
}

\begin{abstract}
Background: Oral cavity and oropharyngeal cancer impose significant threat to airway management. Head and neck radiotherapy (HNRT) may further increase the difficulty of tracheal intubation. We hypothesized that a history of HNRT would be associated with a high rate of difficult tracheal intubation.
\end{abstract}

Methods: Adult patients with a history of HNRT were identified. Non-HNRT controls were case-matched by age, sex and body mass index. The tracheal intubation status between the two patient groups (treated vs. untreated with HNRT) was compared. The $t$ test was used to evaluate differences in continuous variables between the 2 groups. Fisher's exact test or a chi-square test was used to test for associations between radiation status and patient characteristics that may be associated with difficult tracheal intubation. Odds ratio and its confidence interval were used to assess the effect of radiation status on intubation status.

Results: The final cohort of 472 matched patients in age, sex and body mass index consisted of 236 patients who had HNRT before surgery and 236 who had upfront surgery without HNRT. The percentage of patients who had restricted neck range of motion in the HNRT group was significantly higher than in the control group ( $22.3 \%$ vs. $11.0 \% ; p=0.001)$. The proportion of patients with trismus $(p=0.11)$ or difficult tracheal intubation $(p=0.73)$ did not differ significantly between the 2 groups. 12.7\% patients in the study had difficult tracheal intubation. Patients who had mallampati scores of 3 or 4 had significantly higher rate of difficult tracheal intubation than did patients with mallampati scores of 1 or 2 (17.8\% vs. 8.7\%; $p=0.004)$. Multivariate logistic regression model showed no difference between HNRT and intubation status after adjusting neck range of motion and mallampati score (OR $=0.91,95 \% \mathrm{Cl}$ : 0.510 to 1.612 ).

Conclusions: Previous treatment with HNRT was not associated with additional risk of difficult tracheal intubation. Mallampati score may be a sensitive measurement for difficult tracheal intubation in this patient population.

Keywords: Radiotherapy, Cancer, Trismus, Upper aerodigestive track, Airway

\section{Background}

In the United States, an estimated 41,000 oral cavity and oropharyngeal malignancies are diagnosed each year [1]. The anatomic sites of oral cavity cancer (OCC) cover the lips, anterior two-thirds of tongue, gingiva, retromolar trigone, buccal mucosa, hard palate and the floor of mouth; whereas, the sites of oropharyngeal cancer (OPC) are located in the posterior one-third of the

\footnotetext{
* Correspondence: gzheng@mdanderson.org

${ }^{1}$ Department of Anesthesiology and Perioperative Medicine, The University of Texas MD Anderson Cancer Center, 1515 Holcombe Boulevard, Faculty Center - Unit 409, Houston, TX 77030, USA

Full list of author information is available at the end of the article
}

tongue, palatine or lingual tonsils, soft palate, and posterior pharyngeal wall [2].

With the technologic improvement, radiotherapy, depending the subsite of a disease, has become a primary treatment for many head and neck malignancies in order to maximally maintain functionality of the upper aerodigestive track. Approximately $75 \%$ of patients with head and neck squamous cell carcinoma can benefit from head and neck radiation therapy (HNRT) [3, 4]. Although previous receipt of HNRT is a recognized risk factor for difficult airway management, the mechanisms of HNRT- related airway pathologic changes and the

(C) The Author(s). 2019 Open Access This article is distributed under the terms of the Creative Commons Attribution 4.0 International License (http://creativecommons.org/licenses/by/4.0/), which permits unrestricted use, distribution, and 
overall influences of previous HNRT on the outcomes of tracheal intubation remain unclear.

In this retrospective study, we reviewed the performance and outcomes of tracheal intubation in patients who were undergoing surgery to resect primary OCC or OPC of any stage. The primary goal of the study was to determine if previous HNRT adds additional risk to the airway management in the patients with OCC and OPC disregarding the technique applied. In addition, the factors potentially associated with difficult tracheal intubation (DTI) were also analyzed.

\section{Methods}

\section{Patient selection}

The Institutional Review Board (IRB) at The University of Texas MD Anderson Cancer Center approved this study (IRB No. PA12-0699). The informed consent was waived by IRB because this is a retrospective study. A cancer registry including records of 4011 adult (age $\geq 18 \mathrm{y}$ at the time of surgery) patients with primary diagnoses of OCC or OPC who subsequently underwent resection of the primary OCC or OPC tumor at MD Anderson from 2007 to 2012 was used as the primary study database. Patients who underwent tonsillectomy for positive cervical lymphadenopathy with unclear primary disease, whose cases had been used for teaching of flexible endoscopy for tracheal intubation and patients who were $<18$ years of age at the time of HNRT were excluded from this study. In addition, because the majority of the patients in the registry received intensity modulated radiation therapy, those who underwent brachytherapy or proton therapy were excluded in order to ensure the uniformity of the sample.

All the patients in the registry who qualified for the study were assigned into 1 of 2 groups according to whether they had received HNRT: the HNRT group, which consisted of patients who received HNRT before tumor resection, and the non-HNRT group, who underwent upfront resection without having received HNRT. Because some of the data for this study were embedded in the notes describing tracheal intubation in patients' medical records, requiring a manual search, we used a matching strategy to avoid having to hand-search the entire registry. We first identified the HNRT group as described above; then, we used the exact matching method to select matched controls from the non-HNRT group. The control patients were selected according to age, sex, and body mass index (BMI) to match the patients in the HNRT group at a ratio of $1: 1$. The matching range for age was $\pm 5 \mathrm{y}$. For BMI, the 2 groups were matched at 6 levels: $\leq$ $18.5 \mathrm{~kg} / \mathrm{m}^{2}, \quad 18.6-25.0 \mathrm{~kg} / \mathrm{m}^{2}, \quad 25.1-30.0 \mathrm{~kg} / \mathrm{m}^{2}, \quad 30.1-$
$35.0 \mathrm{~kg} / \mathrm{m}^{2}, \quad 35.1-40.0 \mathrm{~kg} / \mathrm{m}^{2}$ and $\geq 40.1 \mathrm{~kg} / \mathrm{m}^{2}$. Each patient in the HNRT group was successfully matched with a non-HNRT control patient.

\section{Data collection}

The following data were electronically retrieved from the patients' medical records: age, sex, BMI, American Society of Anesthesiologists physical status score (ASA score), airway assessment (mouth opening, neck range of motion, edentulous and MP scores), cancer diagnosis, type of surgery, and whether the patient had a history of HNRT. Data on radiotherapy and on the method of tracheal intubation were manually retrieved from the records after matching. The missing data in the primary data sets were manually searched for and placed in the corresponding data set. When a patient had multiple surgeries after HNRT, the data for the first surgery with tracheal intubation after HNRT were used. For airway assessment, most anesthesia providers in our practice considered patients to have trismus if their interincisional distance was $<2$ finger breadths (typically $<$ $3.5 \mathrm{~cm}$ ). However, no standardized criteria were used to measure neck extension, so the neck range of motion was based on providers' subjective judgment. In this study, edentulous referred to a patient with complete upper, lower, or whole-mouth removable dentures. We included the grade of laryngeal view during tracheal intubation in the analysis to reflect the intubation effort according to the Cormack-Lehane system [5]. The grade of laryngeal view mainly applied to the patients who had been intubated via either direct laryngoscopy or video laryngoscopy of any type. For flexible endoscopy trachea intubation, grade I was used for data analysis.

\section{Statistical analysis}

Summary statistics, including mean, standard deviation, median, and range, were calculated for continuous variables, such as age, BMI, and the interval between radiotherapy and surgery. Frequencies and percentages were used to summarize data for categorical variables, such as sex, BMI, Mallampati (MP) score, mouth opening, neck range of motion, cancer stage, radiation status, and airway intubation status. The $t$ test was used to evaluate differences in continuous variables between the 2 patient groups. Fisher's exact test or a chi-square test was used to test for associations between radiation status (HNRT or control) and patient characteristics that may be associated with difficulty of tracheal intubation, including sex, BMI, MP score, mouth opening, neck range of motion, cancer stage, intubation difficulty status (difficult or easy) and patient characteristics. Odds ratio and its confidence interval were used to assess the effect of radiation status on intubation difficulty status. A $P$ value of less than 0.05 was considered statistically significant. 
The statistical software SAS 9.3 (SAS, Cary, NC) was used for all the analyses.

\section{Results \\ Patient characteristics}

Of the 4011 records of eligible patients included in the cancer registry database, 3999 records were eligible for inclusion in this study; 8 patients whose procedures had been used for teaching airway management and 4 patients who had received proton therapy were excluded. The final study cohort of 472 matched patients consisted of 236 patients who had HNRT before surgery and 236 who had upfront surgery without HNRT. The mean $( \pm$ standard deviation) age of the included patients was 58.7 $( \pm 9.1)$ years, and their mean BMI was $25.5( \pm 4.5) \mathrm{kg} /$ $\mathrm{m}^{2}$. The mean interval between completion of HNRT and surgery in the HNRT group was 330.3 ( \pm 474.5) days. Eight primary cancer locations were found in 447 patients. The characteristics of the study cohort are detailed in Table 1.

Data on intubation difficulty status (easy or difficult) were available for 456 of the 472 patients. Among them, $12.7 \%(n=58)$ were described as having had DTI with the corresponding intubation technique used for each case. Intubation with the primary technique failed in 8 patients, whose airways were then managed using either the AirTraq technique $(n=4)$ or asleep intubation with flexible endoscopy $(n=4)$. The airways of $189(40.0 \%)$ patients had been intubated with advanced techniques; the corresponding notes described 6 reasons for use of advanced techniques instead of direct laryngoscopy, including poor mandibular mobility (adequate mouth opening during assessment, but difficulty achieving full mouth opening after anesthetic induction; $n=37$; $19.6 \%)$, cancer growth in the hypopharynx $(n=35$; $18.5 \%$ ), trismus (restricted mouth opening at assessment prior to anesthetic induction, $n=31 ; 16.4 \%)$, distorted airway anatomy from previous surgery $(n=14 ; 7.4 \%)$, short thyromental distance $(n=10 ; 5.3 \%)$, and a large tongue $(n=10 ; 5.3 \%)$. These 6 reasons accounted for $72.5 \%$ of the airways managed by advanced techniques; the reasons for use of advanced airway management techniques in the remaining 52 patients were not specified. Six patients who had initially been scheduled for cancer resection under general anesthesia were found to require tracheostomy before anesthetic induction owing to rapid cancer progression; therefore, airway intubation was not attempted in these patients. No cases of airway loss (cannot intubate and cannot ventilate) were found in the study population.

Radiation status and airway management characteristics The mean ( \pm standard deviation) age and BMI in the HNRT group were $58.3( \pm 9.2)$ y and $25.5( \pm 4.4) \mathrm{kg} /$ $\mathrm{m}^{2}$, respectively. In the control group, the mean age and BMI were $59.1( \pm 9.0)$ y and $25.5( \pm 4.5) \mathrm{kg} / \mathrm{m}^{2}$. The differences in age $(p=0.37)$ and BMI $(p=0.83)$ between the 2 patient groups were not statistically significant. Table 2 shows associations between radiation status and other covariates. The percentage of patients who had restricted neck range of motion in the HNRT group was significantly higher than in the control group $(22.3 \%$ vs. $11.0 \% ; p=0.001)$. In addition, significantly more patients in the HNRT group had advanced-stage cancer than in the control group $(51.2 \%$ vs. $40.8 \% ; p=0.029)$. However, the proportion of patients with trismus $(p=0.11)$ and with DAI $(p=0.73)$ did not differ significantly between the 2 groups. Finally, no significant differences were found between the 2 groups in the grade of laryngeal view during tracheal intubation.

The mean ( \pm standard deviation) time interval between completion of HNRT and surgery was $330 \pm 475$ days and median interval was 134 days. The time interval from HNRT to surgery was not associated with the difficult tracheal intubation $(p=0.9363)$.

\section{Ease of intubation and patient characteristics}

Table 3 displays associations between intubation status and patient characteristics. Data on the intubation status were found for 456 of 472 patients; of these 456 patients, 58 (12.7\%) had DTI. The mean ( \pm standard deviation) age of patients who had DTI was $58.2( \pm 8.8) \mathrm{y}$, and the mean age of patients who did not have DTI was $58.8( \pm$ 9.2) y. The mean BMI of patients with DTI was $24.7( \pm$ 4.3) $\mathrm{kg} / \mathrm{m}^{2}$, and the mean BMI of patients without DTI was $25.6( \pm 4.5) \mathrm{kg} / \mathrm{m}^{2}$. The 2 groups did not differ significantly in age $(p=0.61)$ or in BMI $(p=0.13)$. Patients who had MP scores of 3 or 4 had significantly higher rates of DTI than did patients with MP scores of 1 or 2 (17.8\% vs. $8.7 \%$; $p=0.004)$.

A multivariate logistic regression model was fitted to assess associations between radiation and intubation status after adjustment for neck range of motion and MP score. This analysis revealed no statistically significant association between receiving HNRT and having DTI (Table 4).

\section{Discussion}

The finding in this study did not reveal the correlation between previous HNRT and DTI in patients with OCC or OPC in spite of significant association between HNRT and restriction of neck range of motion. This can be explained by broadly using flexible endoscopy $(35.5 \%)$ for tracheal intubation in the studied population, which effectively overcame the influences of restriction of neck range of motion to the performance of tracheal intubation. Nevertheless, our 
Table 1 Characteristics of 472 patients with oral cavity or oropharyngeal cancer

\begin{tabular}{ll}
\hline Characteristic & \\
\hline Head/neck radiation & \\
No & \\
Yes & \\
Sex & \\
Female & \\
Male & \\
Body mass index $\left(\mathrm{kg} / \mathrm{m}^{2}\right)$ & 176 \\
$\leq 18.5$ & 228 \\
18.6-25.0 & 157 \\
25.1-30.0 & 517 \\
$30.1-35.0$ & 16 \\
$35.1-40.0$ & 3 \\
$\geq 40.1$ &
\end{tabular}

Mallampati score

Data missing a

1

2

3

4

Mouth opening

Data missing

Full

Limited

Thyromental distance $(\approx \mathrm{cm})$

Data missing

$<5$

$\geq 5$

Edentulous Removable denture

Data missing

Yes

No

Neck movement

Data missing

Full range of motion

Restricted

Cancer location

Tongue

Tonsil

Floor of mouth

Mandible

Retromolar region

Buccal mucosa

Maxilla gingiva

No. of patients

236

236

60

412

17

228

157

51
Table 1 Characteristics of 472 patients with oral cavity or oropharyngeal cancer (Continued)

\begin{tabular}{lll}
\hline Characteristic & No. of patients & $\%$ \\
\hline Soft palate & 5 & 1.06 \\
Multiple locations & 25 & 5.30 \\
Cancer stage & & \\
Data missing & 3 & 17.91 \\
T1 & 84 & 33.05 \\
T2 & 155 & 19.62 \\
T3 & 92 & 23.45 \\
VT4 & 110 & 5.12 \\
TX & 24 & 0.85 \\
Not staged & 4 & \\
Intubation status & & 12.72 \\
Data missing & 16 & 87.28 \\
Difficult & 58 & \\
Easy & 398 &
\end{tabular}

Primary intubation technique

\begin{tabular}{|c|c|c|c|}
\hline 13.82 & Data missing & 10 & \\
\hline 44.96 & Direct laryngoscopy & 267 & 57.79 \\
\hline 28.51 & Asleep FOI & 137 & 29.65 \\
\hline \multirow[t]{3}{*}{12.72} & Awake FOI & 27 & 5.84 \\
\hline & AirTraq video laryngoscopy & 15 & 3.25 \\
\hline & C-MAC video laryngoscopy & 7 & 1.52 \\
\hline 78.25 & LMA intubation & 3 & 0.65 \\
\hline \multirow[t]{3}{*}{21.75} & Tracheostomy & 6 & 1.30 \\
\hline & \multicolumn{3}{|l|}{ Secondary intubation technique } \\
\hline & AirTraq video laryngoscopy & 4 & 50 \\
\hline 24.69 & Asleep FOI & 4 & 50 \\
\hline
\end{tabular}

21.44

78.56

83.37

16.63

46.19

20.76

10.38

5.51

5.08

4.66

1.06 finding does not necessarily mean that the influence of HNRT on tracheal intubation should be underestimated. In this study, $22.3 \%$ of patients treated with HNRT had restricted neck movement, whereas only $11.0 \%$ of patients in the non-HNRT group did. In addition, although the difference was not statistically significant, more patients in the HNRT group (24.8\%) than in the non-HNRT group (18.7\%) had trismus. Both restricted neck range of motion and trismus are significant risk factors for difficult direct laryngoscopy. Furthermore, in our clinical experience, trismus and restricted neck range of motion often coexist in patients who develop tissue fibrosis after radiotherapy, posing a significant challenge for both direct 
Table 2 Comparison of patient characteristics by head and neck radiation status

\begin{tabular}{|c|c|c|c|}
\hline Characteristic & Control N (\%) & HNRT N (\%) & $P$ value \\
\hline Sex & & & 1.0 \\
\hline Female & $30(12.7)$ & $30(12.7)$ & \\
\hline Male & $206(87.3)$ & $206(87.3)$ & \\
\hline Body mass index $\left(\mathrm{kg} / \mathrm{m}^{2}\right)$ & & & 1.0 \\
\hline$\leq 18.5$ & $8(3.4)$ & $9(3.8)$ & \\
\hline $18.6-25.0$ & $113(47.9)$ & $115(48.7)$ & \\
\hline $25.1-30.0$ & $79(33.5)$ & $78(33.1)$ & \\
\hline $30.1-35.0$ & $26(11.0)$ & $25(10.6)$ & \\
\hline $35.1-40.0$ & $8(3.4)$ & $8(3.4)$ & \\
\hline$\geq 40.1$ & $2(0.8)$ & $1(0.4)$ & \\
\hline Mallampati score & & & 0.048 \\
\hline 1 & $42(18.3)$ & $21(9.3)$ & \\
\hline 2 & $96(41.7)$ & $109(48.2)$ & \\
\hline 3 & $63(27.4)$ & $67(29.6)$ & \\
\hline 4 & $29(12.6)$ & $29(12.8)$ & \\
\hline Mallampati score (1/2 vs. $3 / 4)$ & & & 0.59 \\
\hline $1 / 2$ & $138(60.0)$ & $130(57.5)$ & \\
\hline $3 / 4$ & $92(40.0)$ & $96(42.5)$ & \\
\hline Mouth opening & & & 0.11 \\
\hline Full & $191(81.3)$ & $176(75.2)$ & \\
\hline Limited & $44(18.7)$ & $58(24.8)$ & \\
\hline Edentulous & & & 0.59 \\
\hline Yes & $53(22.5)$ & $48(20.4)$ & \\
\hline No & $183(77.5)$ & $187(79.6)$ & \\
\hline Neck movement & & & 0.001 \\
\hline Full range & $210(89.0)$ & $181(77.7)$ & \\
\hline Restricted & $26(11.0)$ & $52(22.3)$ & \\
\hline Cancer stage & & & $<0.0001$ \\
\hline $\mathrm{T} 1$ & $28(13.1)$ & $56(24.6)$ & \\
\hline $\mathrm{T} 2$ & $76(35.7)$ & $79(34.6)$ & \\
\hline T3 & $34(16.0)$ & $58(25.4)$ & \\
\hline T4 & $75(35.2)$ & $35(15.4)$ & \\
\hline Intubation status & & & 0.73 \\
\hline Difficult & $31(13.2)$ & $27(12.2)$ & \\
\hline Easy & $203(86.8)$ & $195(87.8)$ & \\
\hline Laryngeal view & & & 0.26 \\
\hline 1 & $80(58.8)$ & $70(49.6)$ & \\
\hline 2 & $41(30.1)$ & $58(41.1)$ & \\
\hline 3 & $10(7.4)$ & $10(7.1)$ & \\
\hline 4 & $5(3.7)$ & $3(2.1)$ & \\
\hline Laryngeal view (1/2 vs. $3 / 4)$ & & & 0.62 \\
\hline $1 / 2$ & $121(89.0)$ & $128(90.8)$ & \\
\hline $3 / 4$ & $15(11.0)$ & $13(9.2)$ & \\
\hline
\end{tabular}

Abbreviation: HNRT head and neck radiation therapy Column percentages are provided laryngoscopy and video laryngoscopy. Because the components of radiation fibrosis syndrome result from the combination of tonic contraction and fibrosis of the muscles of mastication [6], trismus and limited neck range of motion caused by radiation cannot be improved with anesthetic induction or use of a muscle relaxant. This is unlike the trismus induced by cancer pain or inflammation commonly seen in patients who have not undergone radiotherapy, which can often be improved with anesthestics and muscle relaxants. Furthermore, restricted neck range of motion is unlikely to exist in patients without tissue fibrosis unless it is caused by neck-related comorbidities, such as having undergone a neck fusion procedure. No good estimates of the morbidity rate attributable to restriction of neck range of motion after radiotherapy are available, but radiation-induced trismus occurs in up to $45 \%$ of patients who receive curative doses of radiation to the head and neck [7]. Thus, having a back-up plan is important when dealing with airways in patients who have undergone HNRT, so that appropriate tools will be available to manage DTI if it arises.

Another significant finding in this study was that MP scores of 3 or 4 were associated with DTI regardless of the technique used. This result is different from a previous report indicating that MP score alone is not significantly correlated with DTI in patients with otherwise normal airway [8]. The discrepancy can likely be attributed to the study populations. The MP scores in our patients reflected the additive effects of oncopathology, radiation therapy and baseline condition. A previous study reported that up to $75 \%$ of patients developed edema in the head and neck after radiotherapy, and $>50 \%$ of patients had edema in the airway [9]. However, the existing grading systems for assessment of airway edema are unreliable and lack consistency $[10,11]$. Thus, anesthesiologists who manage such patients' airways are unlikely to find clinical reports useful in planning airway management. Patterson et al. [11] developed an airway edema rating scale system by measuring intrarater consistency and interrater agreement on the level of airway edema at several anatomic sites. They found that the aryepiglottic folds and arytenoids were the regions most amenable to visual check (Fiber-laryngoscopy) and that intrarater consistency and interrater agreement were low for the base of the tongue. Because radiotherapy-induced airway edema is mostly diffused throughout the area of treatment, the level of edema found in laryngeal or supraglottic areas may reflect edema in the base of the tongue, pharyngeal well, and floor of the mouth. We speculate that moderate edema in any hypopharyngeal or laryngeal site 
Table 3 Comparison of patient characteristics by intubation

\begin{tabular}{|c|c|c|c|}
\hline Characteristic & DTI N (\%) & Non-DTI N (\%) & $P$ value \\
\hline$\overline{\text { Sex }}$ & & & 0.42 \\
\hline Female & $9(16.1)$ & $47(83.9)$ & \\
\hline Male & $49(12.3)$ & $351(87.8)$ & \\
\hline Body mass index $\left(\mathrm{kg} / \mathrm{m}^{2}\right)$ & & & 0.23 \\
\hline$\leq 18.5$ & $3(17.6)$ & $14(82.4)$ & \\
\hline $18.6-25.0$ & $32(14.7)$ & $185(85.3)$ & \\
\hline $25.1-30.0$ & $19(12.3)$ & $136(87.7)$ & \\
\hline $30.1-35.0$ & $2(4.1)$ & $47(95.9)$ & \\
\hline $35.1-40.0$ & $1(6.7)$ & $14(93.3)$ & \\
\hline$\geq 40.1$ & $1(33.3)$ & $2(66.7)$ & \\
\hline HNRT & & & 0.73 \\
\hline No & $31(13.2)$ & $203(86.8)$ & \\
\hline Yes & $27(12.2)$ & $195(87.8)$ & \\
\hline Mallampati score & & & 0.032 \\
\hline 1 & $4(6.5)$ & $58(93.5)$ & \\
\hline 2 & $19(9.5)$ & $182(90.5)$ & \\
\hline 3 & $22(17.1)$ & $107(82.9)$ & \\
\hline 4 & $11(19.6)$ & $45(80.4)$ & \\
\hline Mallampati score (1/2 vs. $3 / 4)$ & & & 0.004 \\
\hline $1 / 2$ & $23(8.7)$ & $240(91.3)$ & \\
\hline $3 / 4$ & $33(17.8)$ & $152(82.2)$ & \\
\hline Mouth opening & & & 0.53 \\
\hline Full & $44(12.3)$ & $314(87.7)$ & \\
\hline Limited & $14(14.7)$ & $81(85.3)$ & \\
\hline Edentulous & & & 0.42 \\
\hline Yes & $10(10.3)$ & $87(89.7)$ & \\
\hline No & $48(13.4)$ & $310(86.6)$ & \\
\hline Neck movement & & & 0.066 \\
\hline Full range & $44(11.5)$ & $337(88.5)$ & \\
\hline Restricted & $14(19.4)$ & $58(80.6)$ & \\
\hline Cancer stage & & & 0.58 \\
\hline $\mathrm{T} 1$ & $8(9.6)$ & $75(90.4)$ & \\
\hline $\mathrm{T} 2$ & $23(15.1)$ & $129(84.9)$ & \\
\hline $\mathrm{T} 3$ & $10(11.4)$ & $78(88.6)$ & \\
\hline T4 & $11(10.8)$ & $91(89.2)$ & \\
\hline
\end{tabular}

Abbreviations: $D T I$ difficult tracheal intubation, HNRT head and neck radiation therapy

Row percentages are provided combined with an MP score of 3 or 4 is highly suggestive of DTI. Therefore, a bedside flexible endoscopy airway assessment before anesthetic induction on a patient suspected of airway edema should be incorporated into the routine practice for the clinicians who manage high-risk airways.

It is worth mentioning that the intubation technique used in any given case is based not only on clinical indications but also on the personal preference and skill level of the provider. Therefore, the techniques used in the study might not always directly reflect clinical indications. Furthermore, the outcomes of intubation of difficult airways in patients with OCC or OPC are highly experience-dependent. The techniques and equipment used for intubation are also influenced by local culture or practice; similar cases may be managed differently in different institutions $[12,13]$. In this study, the intubations were all performed or supervised by a group of head and neck anesthesiologists who routinely use the techniques described; thus, the results of this study might not be widely applicable to institutions with different practices.

In addition, although the history of HNRT is important to airway management, the anatomic site of the tumor may also affect the airway management. However, the majority of patients (75\%) in this study had tumors with local or regional invasion beyond the primary anatomic sites so that grouping the patients by discrete anatomic margins was not feasible. In addition, these cancers induce tissue reaction or inflammation that often extends the mass effect to a much larger area than the tumor itself. Therefore, the causal relationship between primary cancer site and the airway management was not studied.

\section{Conclusions}

In conclusion, history of HNRT does not increase the overall incidences of DTI in patients with OCC or OPC. Mallampati airway assessment may be used as a sensitive tool to predict difficult tracheal intubation in these patients.

Table 4 Multivariate logistic regression model showing associations between HNRT and intubation status (DAI vs. non-DAI) adjusted for neck range of motion and Mallampati score

\begin{tabular}{llcll}
\hline Effect & $P$ value & Odds ratio estimate & \multicolumn{2}{c}{$95 \% \mathrm{Cl}^{\mathrm{a}}$} \\
Treatment (HNRT vs. Control) & 0.74 & 0.907 & 0.510 & 1.612 \\
\hline Neck motion (restricted vs. full range) & 0.21 & 1.563 & 0.773 \\
Mallampati score (1/2 vs. 3/4) & 0.016 & 0.483 & 0.268 & 3.160 \\
\hline
\end{tabular}

Abbreviations: HNRT head and neck radiation therapy, $\mathrm{Cl}$ confidence interval 


\section{Abbreviations}

ASA score: American Society of Anesthesiologists physical status score; BMI: Body mass index; DTI: Difficult tracheal intubation; HNRT: Head and neck radiation therapy; MP score: Mallampati score; OCC: Oral cavity cancer; OPC: Oropharyngeal cancer

\section{Acknowledgement}

The authors in this article thank A Ninetto, Department of Scientific Publications at MD Anderson Cancer Center for manuscript editing.

\section{Authors' contributions}

G Zheng helped study design, data analysis, manuscript writing and revisions. L Feng helped study design, data analysis and manuscript writing. CM Lewis helped study design, data preparation, manuscript editing and revisions. All authors have read and approved the final manuscript, and ensure that this is the case.

\section{Funding}

None.

\section{Availability of data and materials}

All data generated or analysed during this study are included in this published article and their corresponding figures and tables. Additional data may be available from the corresponding author on reasonable request.

\section{Ethics approval and consent to participate}

The Internal Review Board (IRB) at the University of Texas MD Anderson Cancer Center approved this data review study. The IRB number of the study is PA12-0699.

\section{Consent for publication}

Not applicable.

\section{Competing interests}

The authors declare that they have no competing interests.

\section{Author details}

'Department of Anesthesiology and Perioperative Medicine, The University of Texas MD Anderson Cancer Center, 1515 Holcombe Boulevard, Faculty Center - Unit 409, Houston, TX 77030, USA. ${ }^{2}$ Department of Biostatistics, The University of Texas MD Anderson Cancer Center, FCT4.5047, T. Boone Pickens Academic Tower, 1400 Pressler St, Houston, TX 77030-4008, USA.

${ }^{3}$ Department of Head and Neck Surgery, The University of Texas MD

Anderson Cancer Center, Unit 1445, T. Boone Pickens Academic Tower, 1515

Holcombe Blvd, Houston, TX 77030-4009, USA.

Received: 3 January 2019 Accepted: 24 May 2019

Published online: 01 June 2019

\section{References}

1. Weatherspoon DJ, Chattopadhyay A, Boroumand S, Garcia Al. Oral cavity and oropharyngeal cancer incidence trends and disparities in the United States: 2000-10. Cancer Epidemiol. 2015;39:497-504.

2. Cleveland JL, Junger ML, Saraiya M, Markowitz LE, Dunne EF, Epstein JB. The connection between human papillomavirus and oropharyngeal squamous cell carcinomas in the United States: implications for dentistry. J Am Dent Assoc. 2011;142:915-24.

3. Barton MB, Jacob S, Shafiq J, Wong K, et al. Estimating the demand for radiotherapy from the evidence: a review of changes from 2003 to 2012. Radiother Oncol. 2014;112:140-4.

4. Overgaard J. Improving radiotherapy of squamous cell carcinoma of the head and neck (HNSCC) through a continuous process of biological based clinical trials: a 30 year experience from the Danish head and neck group [AHNS/FHNOS abstract S397]. JAMA Otolaryngol Head Neck Surg. 2014; 140(suppl):S397.

5. Cormack RS, Lehane J. Difficult tracheal intubation in obstetrics. Anaesthesia. 1984:39:1105-11.

6. Wang $\mathrm{CJ}$, Huang EY, Hsu HC, Chen HC, Fang FM, Hsiung CY. The degree and time-course assessment of radiation-induced trismus occurring after radiotherapy for nasopharyngeal cancer. Laryngoscope. 2005;115:1458-60.
7. Rudat V, Dietz A, Nollert J, et al. Acute and late toxicity, tumor control and intrinsic radiosensitivity of primary fibroblasts in vitro of patients with advanced head and neck cancer after concomitant boost radiotherapy. Radiother Oncol. 1999:53:233-45.

8. Shiga T, Wajima Z, Onoue T, Sakamoto A. Predicting difficult intubation in apparently normal patients. Anesthesiology. 2005;103:429-37.

9. Deng J, Ridner SH, Dietrich MS, et al. Prevalence of secondary lymphedema in patients with head and neck cancer. J Pain Symptom Manag. 2012;43:44252.

10. Branski RC, Bhattacharyya N, Shapiro J. The reliability of the assessment of endoscopic laryngeal findings associated with laryngopharyngeal reflux disease. Laryngoscope. 2002;112:1019-24.

11. Patterson JM, Hildreth A, Wilson JA. Measuring edema in irradiated head and neck cancer patients. Ann Otol Rhinol Laryngol. 2007;116:59-564.

12. Mishra S, Bhatnagar S, Jha RR, Sknghal AK. Airway management of patients undergoing oral cancer surgery: a retrospective study. Eur J Anaesthesiol. 2005;22:510-4.

13. Nikhar SA, Sharma A, Ramdaspally M, Gopinath R. Airway management of patients undergoing oral cancer surgery: a retrospective analysis of 156 patients. Turk J Anaesthesiol Reanim. 2017;45:108-11.

\section{Publisher's Note}

Springer Nature remains neutral with regard to jurisdictional claims in published maps and institutional affiliations.

\section{Ready to submit your research? Choose BMC and benefit from:}

- fast, convenient online submission

- thorough peer review by experienced researchers in your field

- rapid publication on acceptance

- support for research data, including large and complex data types

- gold Open Access which fosters wider collaboration and increased citations

- maximum visibility for your research: over $100 \mathrm{M}$ website views per year

At BMC, research is always in progress.

Learn more biomedcentral.com/submissions 